\title{
Relationship between Urinary Pentosidine Concentration and Vascular Complications in Type 2 Diabetic Patients
}

\section{Masami Tanaka*}

Department of Internal Medicine, School of Medicine, Keio University, 35 Shinanomachi, Shinjuku-ku, Tokyo 160-8582, Japan

\begin{abstract}
Objective: I examined the relationship between urinary pentosidine concentration and vascular complications in patients with type 2 diabetes in order to investigate the pathophysiological roles of pentosidine.

Methods: I performed a cross-sectional study in 119 Japanese patients with type 2 diabetes. The correlation of urinary pentosidine concentration with age, diabetes duration, $\mathrm{HbA1c}$ and blood glucose levels was investigated. Urinary pentosidine concentration was compared between patients with and without diabetic microvascular complications (retinopathy, nephropathy, and neuropathy), and macrovascular disease.

Results: Urinary pentosidine concentration showed a significant positive correlation with age and duration of diabetes. However, it did not correlate with $\mathrm{HbA1c}$, fasting plasma glucose, and postprandial plasma glucose. Patients with diabetic retinopathy showed a significantly higher urinary pentosidine concentration than those without retinopathy. On the other hand, urinary pentosidine concentration did not differ between patients with and without nephropathy, neuropathy, and macrovascular diseases. Stepwise regression analysis demonstrated that age, body mass index, and retinopathy were independent determinants of urinary pentosidine concentration. Logistic regression analysis demonstrated that urinary pentosidine concentration and duration of diabetes were independent determinants of diabetic retinopathy.
\end{abstract}

Conclusion: Urinary pentosidine might have significance as a novel biomarker of retinopathy in patients with type 2 diabetes.

Keywords: Advanced glycation end-products (AGEs); Pentosidine, type 2 diabetes mellitus; Diabetic retinopathy; Microvascular complication; Macrovascular complication; Biomarker

\section{Introduction}

Advanced glycation end-products (AGEs) are of great interest in the pathogenesis of both micro- and macrovascular disease. AGEs are formed irreversibly by the sequential non-enzymatic glycation and oxidation of proteins, and form excessive cross-links between tissue proteins [1]. AGEs alter the structure of the extracellular matrix and change the nature of various cells, leading to dysfunction of various organs.

The formation and accumulation of AGEs in the human body occur during normal aging [2]. However, this process is accelerated under a hyperglycemic condition [3]. In diabetic patients, this accelerated formation and accumulation of AGEs might be involved in the development of microvascular complications [4-6].

Pentosidine is one of the best chemically characterized AGE compounds [2,7]. It is known that the concentration of pentosidine increases with age [2], diabetes [8], and renal failure [7,9]. Elevated serum concentrations of pentosidine have been shown in diabetic patients with retinopathy [10-12], nephropathy [13-16], and peripheral artery disease [17]. This phenomenon is observed in both type 1 $[10,16]$ and type $2[11-15,17]$ diabetes. However, the mechanisms by which pentosidine causes micro- and macrovascular complications in diabetic patients are not yet elucidated.

In the present study, I examined the relationship between urinary pentosidine concentration and vascular complications in patients with type 2 diabetes mellitus in order to investigate the pathophysiological roles of pentosidine. In order to compare the importance of pentosidine among micro- and macro- vascular complications, three microvascular diseases (retinopathy, nephropathy, neuropathy) and three macrovascular diseases (cerebral infarction, coronary heart disease, peripheral artery disease) were investigated in the same patients at the same time.

\section{Methods}

\section{Study subjects}

I performed a cross-sectional study in 119 consecutive Japanese patients (60 men and 59 women) with type 2 diabetes mellitus (age 37-85 years; mean 67 years) who were admitted to Keio University Hospital for blood glucose control from January to September 2011. Patients receiving hemodialysis or peritoneal dialysis, whose age was less than 20, who were pregnant, or whose hemodynamic condition was unstable were excluded from this study. The study was approved by the ethical committee of Keio University School of Medicine (IRB approved number: 2010-196) and performed in accordance with the Declaration of Helsinki. Written informed consent was obtained from all subjects.

All patients provided data of their life and medical history, and medication usage at the time of admission. I measured systolic/diastolic blood pressure, height and weight. Blood pressure was measured

${ }^{*}$ Corresponding author: Masami Tanaka, Department of Internal Medicine, School of Medicine, Keio University, 35 Shinanomachi, Shinjuku-ku, Tokyo 160-8582, Japan, Tel: 81-3-5363-3797; Fax: 81-3-3359-2745; E-mail: tana176k@keio.jp

Received January 05, 2015; Accepted January 26, 2016; Published January 30 2016

Citation: Tanaka M (2016) Relationship between Urinary Pentosidine Concentration and Vascular Complications in Type 2 Diabetic Patients. J Diabetes Metab 6: 640 doi:10.4172/2155-6156.1000640

Copyright: ( $) 2016$ Tanaka M. This is an open-access article distributed under the terms of the Creative Commons Attribution License, which permits unrestricted use, distribution, and reproduction in any medium, provided the original author and source are credited. 
during rest in a seated position, measured two times at intervals of $1 \mathrm{~min}$, and the mean value of two measurements was adopted. Body mass index (BMI) was calculated as weight in kilograms divided by height in meters squared. Diabetic microvascular complications (retinopathy, nephropathy, and neuropathy) were precisely evaluated during admission. Diagnosis of diabetic retinopathy was performed by ophthalmologists. Nephropathy was defined as urinary albumin excretion $>30 \mathrm{mg} / \mathrm{g}$ creatinine $(\mathrm{Cr})$ or estimated glomerular filtration rate $(\mathrm{eGFR})<30 \mathrm{ml} / \mathrm{min} / 1.73 \mathrm{~m}^{2}$. Neuropathy was diagnosed with $>2$ of three components: the presence of neuropathic symptoms, the absence of ankle tendon reflexes, and abnormal scores of vibration perception threshold using a C128 tuning fork. Macrovascular disease (cerebral infarction, coronary heart disease, and peripheral artery disease) was defined as a previous history of these diseases. Cerebral infarction includes only ischemic stroke after excluding transient ischemic attack, embolic and hemorrhagic stroke. Coronary heart disease includes unstable angina pectoris, percutaneous coronary intervention, and coronary artery bypass. Hypertension was defined as systolic blood pressure $>140 \mathrm{mmHg}$, diastolic blood pressure $>90 \mathrm{mmHg}$, and/or having received treatment for hypertension. Dyslipidemia was defined as low-density lipoprotein cholesterol (LDL-C) concentration $>120$ $\mathrm{mg} / \mathrm{dl}$, high-density lipoprotein cholesterol (HDL-C) concentration $<40$ $\mathrm{mg} / \mathrm{dl}$, triglyceride $(\mathrm{TG})$ concentration $>150 \mathrm{mg} / \mathrm{dl}$, and/or having received treatment for dyslipidemia. Drinking was defined as prior or current habit of drinking alcohol. Smoking was defined as prior or current tobacco usage.

\section{Laboratory data}

In the morning, after an overnight fast, venous blood samples were collected for measurement of hemoglobin A1c (HbA1c), glucose, hemoglobin, albumin, high-sensitivity C-reactive protein (hs-CRP), $\mathrm{Cr}$, uric acid, LDL-C, HDL-C and TG, and urine samples were collected for measurement of pentosidine and Cr. Venous samples were collected again 2 hours after breakfast for measurement of postprandial plasma glucose. Glucose, Cr, uric acid, LDL-C, HDL-C, and TG were measured by enzymatic method. Hemoglobin and albumin were measured using sodium lauryl sulfate and bromocresol green, respectively. hs-CRP was measured by latex agglutination turbidimetry. Urinary level of pentosidine was measured using high performance liquid chromatography (HPLC) quantification as reported previously $[16,18]$. The intra-assay and inter-assay coefficient of variance in urinary pentosidine measurement were $5.89 \%$ and $8-10 \%$, respectively. The concentration of pentosidine in urine was corrected by the urine concentration of creatinine and expressed as $\mathrm{pmol} / \mathrm{mg} \mathrm{Cr}$. HbAlc was determined by HPLC (Toso, Tokyo, Japan) and presented as the equivalent National Glycohemoglobin Standardization Program (NGSP) value. eGFR was calculated using the following formula established by the working group of the Japanese Chronic Kidney Disease Initiative: eGFR $\left(\mathrm{ml} \mathrm{min}^{-1} 1.73 \mathrm{~m}^{-2}\right)=194 \mathrm{x}$

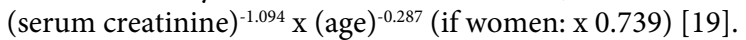

\section{Statistical analysis}

The correlation of urinary pentosidine concentration with age, type 2 diabetes duration, HbAlc, blood glucose levels, BMI, eGFR, uric acid, LDL-C, HDL-C, TG, hemoglobin, serum albumin, and hsCRP. Correlations between two variables were assessed using Pearson's correlations.

Urinary pentosidine concentration was compared between men and women, and between patients with and without hypertension, dyslipidemia, smoking, alcohol drinking, diabetic microvascular complications, and macrovascular disease. Moreover, urinary pentosidine concentration was compared among patients with $0,1,2$, and 3 microvascular complications. Comparisons between two groups and among three or more groups were performed using unpaired t-test and one-way analysis of variance, respectively. Stepwise regression analysis was performed to examine the effects of various factors on urinary pentosidine concentration, and the following factors were considered as independent variables: age, serum albumin, duration of diabetes, retinopathy, and hemoglobin. Logistic regression analysis was performed to examine the effects of various factors on diabetic retinopathy, and the following factors were considered as independent variables: age, urinary pentosidine concentration, $\mathrm{BMI}, \mathrm{HbAlc}$, and duration of diabetes.

All statistical analyses were conducted using the Statistical Package for the Social Sciences (version 17.0; SPSS, Chicago, IL, USA). All data are expressed as mean $\pm \mathrm{SD}$, and values of $p<0.05$ (two-sided) were considered significant.

\section{Results}

As shown in Table 1, the mean of urinary pentosidine concentration was $54.8 \pm 27.1 \mathrm{pmol} / \mathrm{mg}$ Cr. Regarding diabetic microangiopathy, out of 119 patients, 54 (45\%), 48 (40\%), and 68 (57\%) were complicated by retinopathy, nephropathy, and neuropathy, respectively. Regarding macroangiopathy, $14(12 \%), 29(24 \%)$, and $12(10 \%)$ patients were complicated by cerebral infarction, coronary heart disease, and peripheral artery disease, respectively.

Urinary pentosidine concentration showed a significant positive correlation with age $(\mathrm{r}=0.36, \mathrm{p}<0.0001)$ and duration of diabetes $(\mathrm{r}=0.21, \mathrm{p}=0.021)$. On the other hand, correlations of urinary pentosidine concentration with $\mathrm{HbA1c}(\mathrm{r}=-0.020, \mathrm{p}=0.83)$, fasting plasma glucose $(\mathrm{r}=-0.016, \mathrm{p}=0.87)$, and postprandial plasma glucose $(\mathrm{r}=-0.028, \mathrm{p}=0.78)$ were insignificant. Although it showed a significant negative correlation with hemoglobin $(\mathrm{r}=-0.20, \mathrm{p}=0.034)$ and albumin $(\mathrm{r}=-0.34, \mathrm{p}=0.0002)$, correlations with BMI $(\mathrm{r}=-0.090, \mathrm{p}=0.33)$, eGFR $(\mathrm{r}=-0.12, \mathrm{p}=0.18)$ uric acid $(\mathrm{r}=-0.092, \mathrm{p}=0.33)$, LDL-C $(\mathrm{r}=-0.043$, $\mathrm{p}=0.64)$, HDL-C $(\mathrm{r}=-0.10, \mathrm{p}=0.27), \mathrm{TG}(\mathrm{r}=-0.089, \mathrm{p}=0.33)$, and hs-CRP $(\mathrm{r}=-0.057, \mathrm{p}=0.58)$ were insignificant.

\begin{tabular}{|c|c|}
\hline $\mathbf{N}$ (men/women) & $\mathbf{1 1 9} \mathbf{( 6 0 / 5 9 )}$ \\
\hline Age (year-old) & $65.5 \pm 11.6$ \\
\hline Duration of diabetes (years) & $14.6 \pm 9.9$ \\
\hline BMI (kg/m $\left.{ }^{2}\right)$ & $25.2 \pm 5.5$ \\
\hline HbA1c (NGSP) $(\%)$ & $9.4 \pm 2.0$ \\
\hline Urinary pentosidine (pmol/mgCr) & $54.8 \pm 27.1$ \\
\hline Diabetic retinopathy, $\mathrm{n}(\%)$ & $54(45)$ \\
\hline Diabetic nephropathy, $\mathrm{n}(\%)$ & $48(40)$ \\
\hline Diabetic neuropathy, $\mathrm{n}(\%)$ & $68(57)$ \\
\hline Cerebral infarction, $\mathrm{n}(\%)$ & $14(12)$ \\
\hline Coronary heart disease, $\mathrm{n}(\%)$ & $29(24)$ \\
\hline Peripheral artery disease, $\mathrm{n}(\%)$ & $12(10)$ \\
\hline Systolic blood pressure $(\mathrm{mmHg})$ & $127.7 \pm 16.0$ \\
\hline LDL-C $(\mathrm{mg} / \mathrm{dl})$ & $110.6 \pm 30.4$ \\
\hline HDL-C(mg/dl) & $51.6 \pm 16.9$ \\
\hline TG (mg/dl) & $149.6 \pm 112.3$ \\
\hline
\end{tabular}

Table 1: Clinical characteristics of patients in this study. Data presented as mean $\mathrm{SD}$ or $\mathrm{n}(\%)$ of patients. BMI: Body Mass Index; HbA1c: Glycosylated Hemoglobin; LDL-C: Low-density Lipoprotein Cholesterol; HDL-C: High-density Lipoprotein Cholesterol; TG: Triglyceride. 
Citation: Tanaka M (2016) Relationship between Urinary Pentosidine Concentration and Vascular Complications in Type 2 Diabetic Patients. J Diabetes Metab 6: 640. doi:10.4172/2155-6156.1000640

Page 3 of 5

None of gender (men $54.1 \pm 29.0$, women $55.5 \pm 25.2 \mathrm{pmol} / \mathrm{mg}$ $\mathrm{Cr}, \mathrm{p}=0.78$ ), hypertension (absence $53.4 \pm 28.6$, presence $56.8 \pm 28.2$ $\mathrm{pmol} / \mathrm{mg} \mathrm{Cr}, \mathrm{p}=0.53$ ), dyslipidemia (absence $55.4 \pm 24.6$, presence 53.7 $\pm 26.9 \mathrm{pmol} / \mathrm{mg} \mathrm{Cr}, \mathrm{p}=0.76$ ), smoking (absence $51.3 \pm 25.1$, presence $59.7 \pm 29.3 \mathrm{pmol} / \mathrm{mg} \mathrm{Cr}, \mathrm{p}=0.095$ ), and alcohol drinking (absence 52.2 \pm 25.3 , presence $58.6 \pm 29.5 \mathrm{pmol} / \mathrm{mg} \mathrm{Cr}, \mathrm{p}=0.21$ ) showed a significant effect on urinary pentosidine concentration.

As shown in Table 2, patients with diabetic retinopathy showed a significantly higher urinary pentosidine concentration than those without retinopathy. On the other hand, urinary pentosidine concentration did not differ between patients with and without nephropathy, neuropathy, cerebral infarction, coronary artery disease, and peripheral artery disease. As shown in Figure 1, urinary pentosidine concentration was significantly higher in patients with all three diabetic microvascular complications compared with those with none or only one complication.

As shown in Table 3, stepwise regression analysis demonstrated that age $(\beta=0.28, \mathrm{~F}=6.17, \mathrm{p}=0.015), \mathrm{BMI}(\beta=0.27, \mathrm{~F}=5.57, \mathrm{p}=0.020)$ and retinopathy $(\beta=0.24, \mathrm{~F}=4.54, \mathrm{p}=0.036)$ were independent determinants of urinary pentosidine concentration. As shown in Table 4 , logistic regression analysis demonstrated that urinary pentosidine concentration (OR 1.03, 95\% CI 1.01-1.05, $\mathrm{p}=0.0066$ ) and duration of diabetes (OR 1.16, 95\% CI 1.09-1.23, p<0.0001) were independent determinants of diabetic retinopathy.

\section{Discussion}

As AGEs increase with aging and long-term hyperglycemia

\begin{tabular}{|c|c|c|}
\hline \multirow{2}{*}{ Vascular complications } & $\begin{array}{c}\text { Urinary pentosidine } \\
\text { concentration } \\
\text { (pmol/ mg Cr) }\end{array}$ & \multirow{2}{*}{ P } \\
\cline { 2 - 3 } & $63.8 \pm 32.0 / 47.6 \pm 20.0$ & 0.0018 \\
\hline Retinopathy $( \pm /-)$ & $61.0 \pm 33.0 / 50.6 \pm 21.5$ & 0.058 \\
\hline Nephropathy( $\pm /-)$ & $57.5 \pm 29.3 / 51.2 \pm 23.8$ & 0.21 \\
\hline Neuropathy $( \pm /-)$ & $65.3 \pm 32.4 / 53.5 \pm 26.3$ & 0.14 \\
\hline Cerebral infarction( $\pm /-)$ & $54.7 \pm 24.4 / 54.8 \pm 28.1$ & 0.99 \\
\hline Coronary heart disease $( \pm /-)$ & $56.9 \pm 35.9 / 54.6 \pm 26.3$ & 0.79 \\
\hline Peripheral artery disease $( \pm /-)$ & \\
\hline
\end{tabular}

Table 2: Urinary pentosidine concentrations in patients with and without vascular complications. Data presented as mean $\pm \mathrm{SD}$.

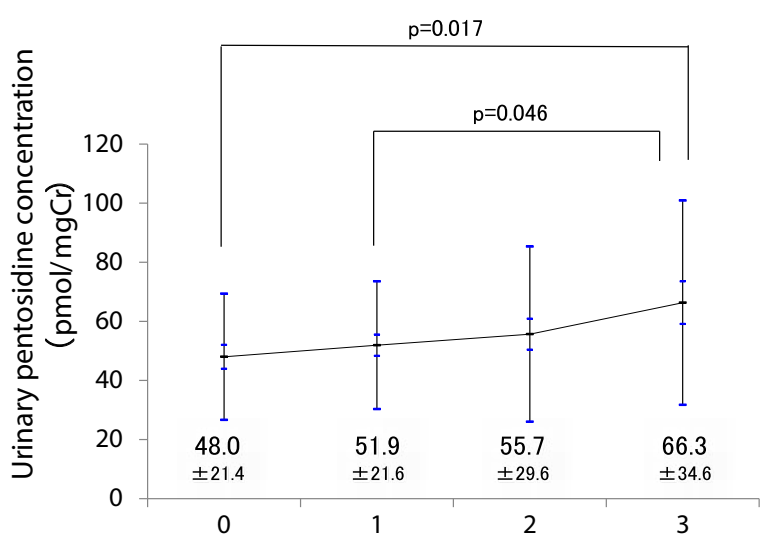

Number of diabetic microvascular complications

Figure 1: Association between urinary pentosidine concentrations and number of diabetic microvascular complications (retinopathy, nephropathy, and neuropathy).

\begin{tabular}{|c|c|c|c|}
\hline variables & $\boldsymbol{\beta}$ & $\mathbf{F}$ & $\mathbf{P}$ \\
\hline Gender & 0.026 & 0.053 & 0.82 \\
\hline Age & 0.28 & 6.17 & 0.015 \\
\hline Serum albumin & -0.16 & 2.52 & 0.12 \\
\hline Duration of diabetes & -0.061 & 0.19 & 0.67 \\
\hline BMI & 0.27 & 5.57 & 0.020 \\
\hline HbA1c & -0.059 & 0.37 & 0.55 \\
\hline eGFR & -0.047 & 0.17 & 0.68 \\
\hline Retinopathy & 0.24 & 4.54 & 0.036 \\
\hline Nephropathy & 0.066 & 0.38 & 0.54 \\
\hline Neuropathy & -0.061 & 0.22 & 0.64 \\
\hline Hypertension & -0.098 & 0.75 & 0.39 \\
\hline Dyslipidemia & -0.027 & 0.063 & 0.80 \\
\hline Smoking & 0.067 & 0.37 & 0.55 \\
\hline Alcohol & 0.096 & 0.69 & 0.41 \\
\hline LDL-C & 0.011 & 0.013 & 0.91 \\
\hline HDL-C & 0.023 & 0.047 & 0.83 \\
\hline TG & -0.14 & 1.66 & 0.20 \\
\hline Hemoglobin & -0.11 & 0.97 & 0.33 \\
\hline
\end{tabular}

BMI: Body Mass Index; HbA1c: Glycosylated Hemoglobin; eGFR: estimated glomerular filtration rate; LDL-C: low-density lipoprotein cholesterol; HDL-C: highdensity lipoprotein cholesterol; TG: triglyceride

Table 3: Stepwise multiple regression analysis to assess independent determinants of urinary pentosidine concentration.

\begin{tabular}{|c|c|c|c|c|}
\hline Variables & Wald & Odds ratio & 95\% Confidence interval & $\mathbf{P}$ \\
\hline Age & 1.61 & 0.97 & $0.93-1.02$ & 0.2 \\
\hline $\begin{array}{c}\text { Urinary pentosidine } \\
\text { concentration }\end{array}$ & 7.37 & 1.03 & $1.01-1.05$ & 0.0066 \\
\hline BMI & 0.0246 & 0.99 & $0.91-1.09$ & 0.88 \\
\hline HbA1c & 0.752 & 1.11 & $0.87-1.42$ & 0.39 \\
\hline Duration of diabetes & 23.03 & 1.16 & $1.09-1.23$ & $<0.0001$ \\
\hline
\end{tabular}

BMI: Body Mass Index; HbA1c: Glycosylated Hemoglobin

Table 4: Multivariate association between selected characteristics and odds ratio of diabetic retinopathy.

accelerates glycation, it is expected that the concentration of pentosidine in blood, urine, or tissues increases in subjects with long-term diabetes. This is supported by the results of my study that the urinary pentosidine concentration correlates with age and diabetes duration. On the other hand, the urinary pentosidine concentration was not correlated with $\mathrm{HbAlc}$, fasting and postprandial plasma glucose levels. This suggests that urinary pentosidine level does not directly reflect the severity of hyperglycemia for at least several months. My finding is consistent with those of Hirata et al. [15], who found that blood pentosidine level did not correlate with $\mathrm{HbAlc}$ in patients with type 2 diabetes, and Sanaka et al. [9], who found that blood pentosidine level did not correlate with blood glucose level. Urinary pentosidine concentration was shown to be negatively correlated with albumin and hemoglobin in this study. Although malnutrition may be involved, the mechanism of this correlation is not clear.

It is expected that urinary pentosidine concentrations of the subjects in this study were high because they were old and their blood glucose control had been poor for a long time, which is reflected by the high prevalence of vascular complications. However, I cannot make this conclusion at present because subjects without diabetes were not included in this study and the reference value of urinary pentosidine concentration in non-diabetic subjects is unknown.

In the present study, it was shown that the urinary concentration 
of pentosidine is higher in patients with diabetic retinopathy than in those without. Moreover, in stepwise multivariate analysis using urinary pentosidine concentration as a dependent variable, age, BMI, and diabetic retinopathy were selected as significant explanatory variables. It is reported that the blood concentration of pentosidine in patients with type 2 diabetic patients increased with the existence and progression of diabetic retinopathy $[11,12]$. However, I could not find any report about the relationship between urinary pentosidine and diabetic retinopathy.

In my study, patients with all three microvascular complications (retinopathy, nephropathy, and neuropathy) had higher pentosidine concentrations compared with patients with no or one complication. There is a report about the relationship between vascular complications and the concentration of AGEs in type 1 diabetic patients with diabetes duration $>50$ years, showing that when the plasma concentrations of both pentosidine and carboxyethyl-lysine, which is another wellcharacterized AGE, are low, the incidence of all three microvascular complications was low [20]. This raised the possibility that pentosidine plays important roles in the onset of microvascular complications in type 1 as well as type 2 diabetes and that there might be common mechanisms underlying the onset of these three microvascular complications [21].

There was no significant difference in urinary pentosidine concentration between patients with and without macrovascular disease in this investigation. However, it was reported that a higher pentosidine concentration was related to a poor outcome of cerebral infarction [22] and cardiac events [20,23], indicating the possibility that pentosidine might play pathophysiological roles, such as induction of oxidative stress and endothelial dysfunction $[24,25]$, in the progression of cardiovascular diseases.

The results of logistic regression analysis showed that urinary pentosidine concentration was an independent explanatory factor for the onset of retinopathy. Combining the results of stepwise regression analysis, it is highly probable that pentosidine might be deeply involved in the onset and progression of diabetic retinopathy, although the mechanism is unclear.

The reason why urinary pentosidine concentration was not associated with diabetic nephropathy, neuropathy, and macrovascular diseases in the present study is not clear. One possibility is that other than common mechanisms by which pentosidine induces three microvascular and three macrovascular complications, there may exist certain mechanisms by which pentosidine induces retinopathy specifically. In addition to circulating pentosidine, local pentosidine in retina might play important roles. It is reported that AGEs are localized in retinal blood vessels [26] or in aqueous humor in type 2 diabetic patients, and its expression correlates with the severity of retinopathy $[27,28]$. These observations indicate the possibility that not only circulating AGEs but also localized AGEs might play important pathophysiological roles. It is reported that infused AGEs crossed the blood retinal barrier in rats [29]. In vitro studies also showed that when retinal cells were exposed to AGEs, their expression of vascular endothelial growth factor increased, which might promote retinal neovascularization and increase permeability of the retinal barrier [30].

Most previous reports investigated the relationship between diabetic complications and serum or tissue $[31,32]$ pentosidine level. On the contrary, I investigated urinary pentosidine. It is reported that the concentrations of both serum and urinary pentosidine are high in patients with active rheumatoid arthritis [33] and elderly patients with cerebral infarction [34]. It is also reported that urinary pentosidine level was correlated with serum pentosidine level, indicating that circulating pentosidine in the blood might be excreted into the urine according to its serum level [34]. As urine collection is less invasive than blood or tissue collection, urinary pentosidine is expected to be a useful tool for the evaluation of diabetic vascular complications.

Limitations of this study include its cross-sectional design. In addition, patients admitted to university hospitals are selected patients, and the results of my study may not be applicable to the general population or to patients with type 2 diabetes in a primary care setting. Furthermore, subjects without diabetes were not included in my study, indicating that my study had no control.

Although I am unable to clarify the cause and effect relationship between pentosidine and diabetic vascular complications, my findings suggest that urinary pentosidine might have significance as a novel biomarker of retinopathy in patients with type 2 diabetes. It remains to be clarified whether measurement of urinary pentosidine concentration will help prevent the onset or progression of vascular complications. Further prospective studies will be necessary to address these issues.

\section{References}

1. Brownlee M (1995) Advanced protein glycosylation in diabetes and aging. Ann Rev Med 46: 223-234.

2. Sell DR, Monnier V (1989) Structure elucidation of a senescence cross-link from human extracellular matrix: implication of pentosis in the ageing process. J Biol Chem 264: 21597-32602.

3. Brownlee M, Cerami A, Vlassara H (1988) Advanced glycosylation end products in tissue and the biochemical basis of diabetic complications. N Eng J Med 318: 1315-1321

4. Besswenger PJ, Makita Z, Curphery TJ, Moore LL, Jean S, et al. (1995) Formation of immunochemical advanced glycosylation end products precedes and correlates with early manifestations of renal and retinal disease in diabetes. Diabetes 44: 824-829.

5. Yamagishi S, Fujimori H, Yonekura H, Yamamoto Y, Yamamoto H (1998) Advanced glycation endproducts inhibit prostacyclin production and induce plasminogen activator inhibitor-1 in human microvascular endothelial cells. Diabetologia 41: 1435-1441.

6. McCance DR, Dyer DG, Dunn JA, Bailie KE, Thorpe SR, et al. (1993) Maillard reaction products and their relation to complications in insulin-dependent diabetes mellitus. J Clin Invest 91: 2470-2478.

7. Sell DR, Nagaraj RH, Grandhee SK, Odetti P, Lapolla A, et al. (1991) Pentosidine: a molecular marker for the cumulative damage to proteins in diabetes, aging, and uremia. Diabetes Metab Rev 7: 239-251.

8. Daimon M, Sugiyama K, Kameda W, Saitoh T, Oizumi T, et al. (2003) Increased urinary levels of pentosidine, Pyrraline and acrolein adduct in type 2 diabetes. Endocr J 50: 61-67.

9. Sanaka T, Funaki T, Tanaka T, Hoshi S, Niwayama J, et al. (2002) Plasma pentosidine levels measured by a newly developed method using ELISA in patients with chronic renal failure. Nephron 91: 64-73.

10. Salman AG, Mansour DE, Swelem AH, Al-Zawahary WM, et al. (2009) Pentosidine - a new biochemical marker in diabetic retinopathy. Ophthalmic Res 42: 96-98.

11. Ghanem AA, Elewa A, Arafa LF (2011) Pentosidine and N-carboxymethyllysine: biomarkers for type 2 diabetic retinopathy. Eur $\mathrm{J}$ Ophthalmol 21: 48-54.

12. Kerkeni M, Saidi A, Bouzidi H, Letaief A, Ben Yahia S, et al. (2013) Pentosidine as a biomarker for microvascular complications in type 2 diabetic patients. Diab Vasc Dis Res 10: 239-245.

13. Piarulli F, Sartore G, Ceriello A, Ragazzi E, Reitano R, et al. (2009) Relationship between glycol-oxidation, antioxidant status and microalbuminuria in type 2 diabetic patients. Diabetologia 52: 1419-1425.

14. Gohda T, Tanimoto M, Moon JY, Gotoh H, Aoki T, et al. (2008) Increased serum 
Citation: Tanaka M (2016) Relationship between Urinary Pentosidine Concentration and Vascular Complications in Type 2 Diabetic Patients. J Diabetes Metab 6: 640. doi:10.4172/2155-6156.1000640

Page 5 of 5

endogenous secretory receptor for advanced glycation end-product (esRAGE) levels in type 2 diabetic patients with decreased renal function. Diabetes Res Clin Pract 81: 196-201.

15. Hirata K, Kubo K (2004) Relationship between blood levels of N-carboxymethyllysine and pentosidine and the severity of microangiopathy in type 2 diabetes. Endocrin J 51: 537-544

16. Tsukahara H, Sekine K, Uchiyama M, Kawakami H, Hata I, et al. (2003) Formation of advanced glycosylation end products and oxidative stress in young patients with type 1 diabetes. Pediatr Res 54: 419-424.

17. Lapolla A, Piarulli F, Sartore G, Ceriello A, Ragazzi E, et al. (2007) Advanced glycation end products and antioxidant status in type 2 diabetic patients with and without peripheral artery disease. Diabetes Care 30: 670-676.

18. Tsukahara H, Ohta N, Sato S, Hiraoka M, Shukunami K, et al. (2004) Concentration of pentosidine, an advanced glycation end-product, in umbilical cord blood. Free Radic Res 38: 691-695

19. Matsuo S, Imai E, Horio M, Yasuda Y, Tomita K, et al. (2009) Revised equations for estimated GFR from serum creatinine in Japan. Am J Kidney Dis 53: 982 992.

20. Sun JK, Keenan HA, Cavallerano JD, Asztalos BF, Schaefer EJ et al. (2011) Protection from retinopathy and other complications in patients with type 1 diabetes of extreme duration: the Joslin 50-year medalist study. Diabetes Care 34: $968-974$

21. Goh SY, Cooper ME (2008) The role of advanced glycation end products in progression and complications of diabetes. J Clin Endocrinol Metab 93: 1114311152.

22. Ikeda T, Maruyama K, Ito N, Utagawa A, Nagane M, et al. (2012) Serum pentosidine, an advanced glycation end product, indicates poor outcomes after acute ischemic stroke. J Stroke Cerebrovasc Dis 21: 386-390.

23. Koyama Y, Takeishi Y, Arimoto T, Niizeki T, Shishido T, et al. (2007) High serum level of pentosidine, an advanced glycation end product (AGE), is a risk factor of patients with heart failure. J Card Fail 13: 199-206.

24. Wautier JL, Schmidt AM (2004) Protein glycation: A firm link to endothelial cell dysfunction. Circ Res 95: 233-238.
25. Basta G, Schmidt AM, De Caterina R (2004) Advanced glycation end products and vascular inflammation: Implications for accelerated atherosclerosis in diabetes. Cardiovasc Res 63: 582-592.

26. Murata T, Nagai R, Ishibashi T, Inomuta H, Ikeda K, et al. (1997) The relationship between accumulation of advanced glycation end products and expression of vascular endothelial growth factor in human diabetic retinas. Diabetologia 40: 764-769.

27. Stitt AW (2001) Advanced glycation: an important pathophysiological event in diabetic and age related ocular disease. $\mathrm{Br} \mathrm{J}$ Ophthalmol 85: 746-753.

28. Endo M, Yanagisawa K, Tsuchida K, Okamoto T, Matsushita T, et al. (2001) Increased levels of vascular endothelial growth factor and advanced glycation end products in aqueous humor of patients with diabetic retinopathy. Horm Metab Res 33: 317-322.

29. Stitt AW, Li YM, Gardiner TA, Bucala R, Archer DB, et al. (1997) Advanced glycation end products (AGEs) co-localize with AGE receptors in the retina vasculature of diabetic and of AGE-infused rats. Am J Pathol 150: 523-531.

30. Lu M, Kuroki M, Amano S, Tolentino M, Keough K, et al. (1998) Advanced glycation end products increases retinal vascular endothelial growth factor expression. J Clin Invest 101: 1219-1224.

31. Beisswenger PJ, Moore LL, Brinck-Johnsen T, Curphey TJ (1993) Increased collagen-linked pentosidine levels and advanced glycation end products in early diabetic nephropathy. J Clin Invest 92: 212-217.

32. Monnier VM, Bautista O, Kenny D, Sell DR, Fogarty J, et al. (1999) Skin collagen glycation, glycoxidation, and crosslinking are lower in subjects with long term intensive versus conventional therapy of type 1 diabetes. Relevance of glycated collagen products versus $\mathrm{HbA} 1 \mathrm{c}$ as markers of diabetic complications. Diabetes 48: 870-880.

33. Takahashi M, Suzuki M, Kushida K, Miyamoto S, Inoue T. (1997) Relationship between pentosidine levels in serum and urine and activity in rheumatoid arthritis. Br J Rheumatol 36: 637-642.

34. Yoshihara K, Nakamura K, Kanai M, Nagayama Y, Takahashi S, et al. (1998) Determination of urinary and serum pentosidine and its application to elder patients. Biol Pharm Bull 21: 1005-1008. 